\title{
Gene Families of AmpC-producing Enterobacteriaceae Present in the Intensive Care Unit of Cipto Mangunkusumo Hospital Jakarta
}

\author{
Lucky Hartati Moehario ${ }^{1, *}$, Thomas Robertus ${ }^{1}$, Anis Karuniawati², Rudyanto Sedono ${ }^{3}$, \\ Delly Chipta Lestari ${ }^{2}$, Andi Yasmon ${ }^{2}$ \\ ${ }^{1}$ Department of Microbiology, Faculty of Medicine and Health Science, Atma Jaya Catholic University of Indonesia, Jl. Pluit Raya No. 2, \\ Jakarta, Indonesia \\ ${ }^{2}$ Department of Microbiology, Faculty of Medicine, Universitas Indonesia, Jl. Salemba Raya No. 4, Jakarta, Indonesia \\ ${ }^{3}$ Intensive Care Unit, Cipto Mangunkusumo Hospital, Jl. Diponegoro No. 71, Jakarta, Indonesia \\ *Corresponding author. E-mail: luckyhmoehario@gmail.com
}

Received date: Sep 12, 2018; Revised date: Dec 7, 2018; Accepted date: Dec 19, 2018

\section{Abstract}

$\mathrm{B}$ ACKGROUND: Antibiotic resistance has become a worldwide problem. Among Asia countries, Indonesia has high prevalence of multi-drug resistant organisms mainly due to Gram-negative bacilli Enterobacteriaceae. This study aimed to find out whether gene family of AmpC and AmpC/ESBL-producing Enterobacteriaceae were present in the Intensive Care Unit (ICU) of Cipto Mangunkusumo Hospital, Jakarta, Indonesia.

METHODS: Specimens were obtained from several body sites of adult patients with infection hospitalised in ICU of Cipto Mangunkusumo Hospital. VITEK®2 was used to identify the microorganisms. Antibiotic susceptibility tests were conducted using VITEK ${ }^{\circledR} 2$ and disc diffusion technique according to Clinical and Laboratory Standards Institute (CLSI) guidelines. Double disc synergy (DDS) test method was employed to detect AmpC activity. Gene families of $\operatorname{ampC}$ were identified using multiplex polymerase chain reaction (PCR).

\section{Introduction}

Antimicrobial resistance has become a serious problem worldwide, especially in Asia.(1) Indonesia is one of the Asian countries where the prevalence of multi drugresistant organisms (MDRO) is very high. The high resistance rates is mainly found in Gram-negative bacteria
RESULTS: Forty five isolates were identified as putative AmpC, extended-spectrum $\beta$-lactamases (ESBL) and AmpC/ESBL-producing Enterobacteriaceae. Klebsiella pneumoniae $(\mathrm{n}=32)$ were predominant, followed by Escherichia coli $(\mathrm{n}=6)$, Enterobacter cloacae $(\mathrm{n}=5)$ and Enterobacter aerogenes $(\mathrm{n}=2)$. AmpC activity was detected in 9 isolates, in which 4 isolates were $\mathrm{AmpC}$ producing and 5 isolates were AmpC/ESBL. In vitro, AmpC-producing Enterobacteriaceae showed good susceptibility to many antibiotic tested, while those of AmpC/ESBL-producing only to Amikacin. The gene families of $\operatorname{ampC}$ were DHA, $\mathrm{EBC}$ and CIT identified from 6 isolates.

CONCLUSION: DHA, EBC and CIT gene families were identified from AmpC and AmpC/ESBL-producing Enterobacteriaceae in the ICU of Cipto Mangunkusumo Hospital. While the AmpC-producing was still susceptible to almost all antibiotics tested, the AmpC/ESBL-producing showed resistant except for Amikacin.

KEYWORDS: Enterobacteriaceae, $\beta$-lactamases, AmpC, ESBL

Indones Biomed J. 2019; 11(1): 107-12 such as Pseudomonas aeruginosa, Enterobacteriaceae and Acinetobacter baumannii. The same situation applies in animal farms.(2) The resistance rates of Klebsiella pneumoniae, Klebsiella ozaenae and Escherichia coli isolated from Intensive Care Unit (ICU) of Fatmawati Hospital were $75.7 \%, 81.5 \%$ and $46.2 \%$ to Ceftriaxone; $67.9 \%, 100 \%$ and $46.2 \%$ to Cefotaxime; $73 \%, 85.7 \%$ and $38.5 \%$ to Ceftazidime.(3) In the ICU of Cipto 
Mangunkusumo Hospita, Jakarta, however, the prevalence of carbapenem resistant i.e., Enterobacteriaceae 27.6\%, P. aeruginosa $21.9 \%$ and $A$. baumannii $50.5 \%$.(4)

The major mechanism of resistance of Gram-negative bacteria is from the production of $\beta$-lactamases, such as AmpC $\beta$-lactamases (AmpC) and extended spectrum $\beta$-lactamases (ESBL).(5) The AmpC producers confer resistance to penicillins, cephamycins (i.e., Cefoxitin, Cefotetan), oxyimino-cephalosporins (i.e., Ceftazidime, Cefotaxime, Ceftriaxone) and monobactams (i.e., Aztreonam). This type of microorganism shows resistant to antibiotic combination of $\beta$-lactam and $\beta$-lactamase inhibitors i.e., Amoxicillin/Clavulanic acid. The AmpC $\beta$-lactamases are chromosomal-mediated, and many Gram-Negative bacteria such as Enterobacter spp., Citrobacter spp., Serratia spp., Morganella morganii, Aeromonas spp. and Hafnia alvei have been known to produce AmpC $\beta$-lactamases. $(6,7,8)$ The widely use of $\beta$-lactam antibiotics and $\beta$-lactamase inhibitors induces a high level expression of chromosomalmediated AmpC and causes the resistant to among other the $3^{\text {rd }}$ generation of cephalosporin and carbapenem. In hospitals where $3^{\text {rd }}$ generation cephalosporins are being used repeatedly for a long period of time, the problem of antibiotic resistance arise. Enterobacter aerogenes and Enterobacter cloacae which are initially susceptible to $3^{\text {rd }}$ generation cephalosporins have become resistant upon therapy.(8)

Apart from the chromosomal gene, the $\mathrm{AmpC}$ is also encoded by the plasmid. Gram-negative bacteria such as Klebsiella oxytoca, Proteus mirabilis, Citrobacter freundii and Enterobacter aerogenes are among those with plasmid-mediated AmpC. $(8,9,10,11)$ Differ from chromosomal-mediated AmpC, plasmid-mediated AmpC are expressed significantly, and are typically associated with broad multidrug resistance. $(10,11)$ The microorganisms that are over expressing $\mathrm{AmpC}$ can become resistant to carbapenem when there is disturbance of the outer membrane permeability.(6) At present, 6 types of plasmidmediated $a m p C$ gene family has been found, namely DHA (firstly isolated at Dhahran Hospitals in Saudi Arabia), EBC (isolated from Enterobacter cloacae), CIT (firstly isolated from Citrobacter freundii), Ambler class C (ACC), FOX (Active on Cefoxitin), and MOX (Active on Moxalactam).

In the past decades, the prevalence of AmpCproducing Enterobacteriaceae had increased, and resulted in a serious threat, among other these bacteria are the major cause of hospital-acquired infections.(12,13) However, the information regarding AmpC-producing Enterobacteriaceae originated from hospitalised patients in Indonesia is not available thus far. This study aimed to explore the presence of AmpC- $\beta$-lactamase producing Enterobacteriaceae gene family in the ICU of Cipto Mangunkusumo Hospital, Jakarta, and also their antibiotic susceptibility.

\section{Methods}

This was a descriptive and cross sectional study. Clinical specimens i.e., blood, lower respiratory tract secretions, urine, wound swabs, pus and soft tissues obtained from adult patients with infection in the ICU of Cipto Mangunkusumo Hospital within 6 months from April to September 2015 were subjected for the investigation. Bacterial cultivation workup was performed in the Clinical Microbiology laboratory of the Department of Microbiology, Faculty of Medicine, Universitas Indonesia. All patients that agreed to give their specimens were enrolled in this study, and signed informed consent.

\section{Bacterial Isolates}

Bacterial identification was conducted using VITEK ${ }^{\circledR} 2$ (Bio-Mérieux, Craponne, France). Anti-biotic susceptibility tests were conducted using VITEK $® 2$ for Ampicillin, Gentamicin, To-bramycin, Amikacin, Ampicillin/ Sulbactam, Piperacillin/Tazobactam, Cefoxitin, Cefotaxime, Ceftazidime, Cefepime, Meropenem, Ciprofloxacin, Levofloxacin, Fosfomycin, Tetracycline, Cotrimoxazole. As for Ceftriaxone, Cefpodoxime, and Aztreonam, disc diffusion technique was employed according to Clinical and Laboratory Standards Institute (CLSI) 2014 guidelines. (14) The antibiotic activity was classified as good if the susceptibility of all isolates to the antibiotic was $80 \%$ or greater. Isolates which less susceptible or resistant to one or more of $3^{\text {rd }}$ generation cephalosporins (i.e., Cefpodoxime, Ceftazidime, Cefotaxime, Ceftriaxone) and/or Aztreonam and/or resistance to Cefoxitin were considered as putative AmpC $\beta$-lactamases, ESBL or AmpC/ESBL producers, and were included in this study. These isolates underwent confirmation test i.e., double disc synergy test (DDS) for AmpC $\beta$-lactamases and ESBL and subjected to multiplex polymerase chain reaction (PCR) for the detection of ampC gene families.

\section{Confirmation Tests for AmpC $\beta$-lactamases and ESBL Using DDS Test}

DDS test method using Total ESBL+AmpC kit 98019 (ROSCO Diagnostica, Taastrup, Denmark) was employed to detect AmpC and ESBL activity. The assay was performed 
according to recommendations in the user manual. Antibiotic discs used were Cefotaxime + Cloxacillin (CTXCX), Cefotaxime + Clavulanate (CTXC), Cefotaxime + Clavulanate + Cloxacillin (CTXCC), Ceftazidime + Cloxacillin (CAZCX), Ceftazidime + Clavulanate (CAZC) and Ceftazidime + Clavulanate + Cloxacillin $($ CAZCC). Isolates were confirmed as AmpC-producing when the inhibition zone produced around CTXC/CTXCC and/ or CAZC/CAZCC discs was differ $\geq 5 \mathrm{~mm}$. An ESBLproducing was confirmed if the inhibition zone produced around the CTXCX/CTXCC and/or CAZCX/CAZCC discs was $\geq 5 \mathrm{~mm}$. If the inhibition zones produced around CTXCX/CTXCC and the CTXC/CTXCC discs were differ $\geq 5 \mathrm{~mm}$, and/or the CAZCX/CAZCC and CAZC/CAZCC discs were $\geq 5 \mathrm{~mm}$ then they were confirmed as producing both AmpC/ESBL enzymes. Escherichia coli ATCC 25922 was used as quality control strain.

The proportion of AmpC and AmpC/ESBL-producing Enterobacteriaceae was determined by dividing the number of those obtained from DDS test with all putative isolates which were less susceptible or resistant to one or more of $3^{\text {rd }}$ generation cephalosporins and/or Aztreonam and/or resistance to Cefoxitin.

\section{Multiplex PCR for the Identification of AmpC Gene Families}

The DNA extraction was performed from fresh culture using boiling techniques. Five to 10 colonies of overnight bacterial culture were suspended into $500 \mu \mathrm{L}$ of sterile phosphate buffer saline (PBS), mixed on a vortex mixer, and spin at $12,000 \mathrm{rpm}$ for $2 \mathrm{~min}$ (2 times). After the supernatant was decanted, the pellet was resuspended in $200 \mu \mathrm{L}$ of TE buffer and the mixture was briefly mixed on a vortex mixer. The cells were lysed by heating at $90^{\circ} \mathrm{C}$ for $20 \mathrm{~min}$ and cellular debris was removed by centrifugation at $12,000 \mathrm{rpm}$ for 10 $\min$. A $100 \mu \mathrm{L}$ aliquot of the supernatant was transferred to a sterile tube and stored at $-20^{\circ} \mathrm{C}$ until PCR testing. Multiplex PCR (Philisa ${ }^{\circledR}$ ampC ID Kit, Streck, Inc., La Vista, USA) was conducted to detect 6 different families of plasmidmediated ampC genes i.e., ACC, CIT, DHA, EBC, FOX and MOX, and primers were used as published earlier.(7) The assay was performed according to recommendations in the user manual.

\section{Ethical Clearance}

This study has passed ethical evaluations by the Faculty of Medicine, Universitas Indonesia No. 51/UN2.F1/ ETIK/2015 and Cipto Mangunkusumo Hospital Ethics Committee No. LB.02.01/X.2/105/2015.

\section{Results}

A total of 370 clinical specimens were collected from patients with infections in the ICU of Cipto Mangunkusumo Hospital from April 2015 to September 2015. After the cultivation and bacterial identification processes 63 isolates were identified as Enterobacteriaceae, and tested further for antibiotic susceptibility. The results showed 45 isolates composed of $K$. pneumoniae, E. coli, E. cloacae and E. aerogenes with decreased susceptibility to one or more of the $3^{\text {rd }}$ generation cephalosporins and/or Aztreonam and/or resistance to Cefoxitin. Among those 4 species, Klebsiella pneumoniae was the most prominent (71.1\%) and sputum was the main source of isolates $(75.6 \%)$ (Table 1$)$.

The DDS test was employed to these 45 isolates and the results were shown in Table 1, as follow: Four isolates were the AmpC-producing Enterobacteriaceae i.e., $K$. pneumoniae $(\mathrm{n}=1)$, E. cloacae $(\mathrm{n}=2)$, and E. aerogenes $(\mathrm{n}=1)$. Thirty three isolates were the ESBL-producers in which $K$. pneumoniae was the most prominent among other Enterobactericeae. Five isolates were the AmpC/

Table 1. Species bacteria isolated, type of specimen and AmpC-producing Enterobacteriaceae determined by DDS test taken from patients in the ICU of Cipto Mangunkusumo Hospital.

\begin{tabular}{|c|c|c|c|c|c|c|c|c|c|c|}
\hline \multirow[b]{2}{*}{ Bacteria } & \multicolumn{6}{|c|}{ Specimen Types (\%) } & \multicolumn{4}{|c|}{ Double Disc Synergy Test } \\
\hline & Sputum & $\begin{array}{c}\text { Wound } \\
\text { Swab }\end{array}$ & Blood & Urine & $\begin{array}{l}\text { Abscess } \\
\text { Aspirate }\end{array}$ & $\begin{array}{l}\text { Total, } \\
\text { n (\%) }\end{array}$ & AmpC & ESBL & $\begin{array}{l}\text { AmpC } \\
\text { ESBL }\end{array}$ & $\begin{array}{c}\text { Non- } \\
\text { producing }\end{array}$ \\
\hline$K$. pneumoniae & 27 & 3 & 2 & 0 & 0 & $32(71.1)$ & 1 & 27 & 2 & 2 \\
\hline E. coli & 2 & 1 & 0 & 2 & 1 & $6(13.4)$ & 0 & 5 & 1 & 0 \\
\hline E. cloacae & 4 & 1 & 0 & 0 & 0 & $5(11.1)$ & 2 & 0 & 2 & 1 \\
\hline E. aerogenes & 1 & 0 & 1 & 0 & 0 & $2(4.4)$ & 1 & 1 & 0 & 0 \\
\hline Total, n (\%) & 34 (75.6) & $5(11.1)$ & $3(6.7)$ & $2(4.4)$ & $1(2.2)$ & $45(100)$ & 4 & 33 & 5 & 3 \\
\hline
\end{tabular}


ESBL producers i.e., K. pneumoniae $(\mathrm{n}=2)$, E. cloacae $(\mathrm{n}=2)$ and $E$. coli $(\mathrm{n}=1)$. Three isolates were non-producing $\beta$-lactamases. In brief, out of 45 isolates of the putative AmpC $\beta$-lactamases and/or ESBL producers, only $9(20 \%)$ isolates were confirmed by DDS test as the AmpC and AmpC/ESBL producers.

Antibiotic susceptibility tests were carried out as mentioned in the methods for the $42 \mathrm{AmpC}$, ESBL, and AmpC/ESBL confirmed isolates. Three isolates of the non-producing $\beta$-lactamases were not tested. As shown in Tables 2, the AmpC-producing isolates (4 isolates) showed $\geq 75 \%$ susceptibility to Gentamicin, Tobramycin, Amikacin, Cefepime, Meropenem, Ciprofloxacin, Levofloxacin, Tetracycline and Cotrimoxazole. All 5 isolates of the AmpC/ ESBL-producing isolates showed resistant to all antibiotics but to Amikacin.

Gene family of AmpC-producing Enterobacteriaceae was determined by multiplex PCR on all of 45 isolates (Figure 1). The results showed that $a m p C$ genes were detected in 6 isolates consisted of 4 species i.e., $K$. pneumoniae, E. cloacae, E. aerogenes and E. coli. The ampC gene families, i.e., DHA, EBC and CIT were identified. DHA gene family was identified from 2 isolates of $K$. pneumoniae and 1 isolate of E. cloacae. EBC was from 1 isolate each of E. cloacae and of E. aerogenes, and lastly CIT was from 1 isolate of E. coli (Table 3).

\section{Discussion}

This study demonstrates that gene families of AmpCproducing Enterobacteriaceae are present in the ICU of Cipto Mangunkusumo Hospital, Jakarta. The distribution of the total proportion of AmpC-producing Enterobacteriaceae was $20 \%$, consisting of 9 isolates out of 45 putative putative AmpC $\beta$-lactamases and/or ESBL producers. The result was quite similar with previous studies carried in India, Singapore and South Korea. $(8,9,12,15)$ Our study showed that among 9 isolates of the AmpC producers, 5 of them produced $\mathrm{AmpC}$ and ESBL. Unlike the AmpC/ESBL producers that showed resistant to all antibiotics except for Amikacin, the AmpCproducing isolates were susceptible to many antibiotics such as Gentamicin, Tobramycin, Amikacin, Cefepime, Meropenem, Ciprofloxacin, Levofloxacin, Tetracycline, and Cotrimoxazole. In this study, 1 of the AmpC-producing isolate and $3 \mathrm{AmpC} / \mathrm{ESBL}-$ producing isolates showed

Table 2. Antimicrobial susceptibility patterns of the AmpC, ESBL and both AmpC/ESBL producing Enterobacteriaceae.

\begin{tabular}{|c|c|c|c|}
\hline Antibiotic & $\begin{array}{c}\operatorname{AmpC}(\%) \\
(n=4)\end{array}$ & $\begin{array}{c}\text { ESBL }(\%) \\
(n=33)\end{array}$ & $\begin{array}{c}\operatorname{AmpC} / \operatorname{ESBL}(\%) \\
(\mathrm{n}=5)\end{array}$ \\
\hline \multicolumn{4}{|l|}{ Beta-lactams } \\
\hline Ampicillin & 0 & 0 & 0 \\
\hline Ampicillin/sulbactam & 0 & 0 & 0 \\
\hline Piperacillin/tazobactam & 25 & 42 & 20 \\
\hline Cefoxitin & 0 & 58 & 0 \\
\hline Cefotaxime & 25 & 0 & 0 \\
\hline Ceftriaxone & 25 & 0 & 0 \\
\hline Cefpodoxime & 25 & 0 & 0 \\
\hline Ceftazidime & 25 & 0 & 0 \\
\hline Cefepime & 75 & 0 & 20 \\
\hline Meropenem & 75 & 73 & 40 \\
\hline Aztreonam & 50 & 9 & 0 \\
\hline \multicolumn{4}{|l|}{ Quinolones } \\
\hline Ciprofloxacin & 75 & 9 & 0 \\
\hline Levofloxacin & 75 & 15 & 20 \\
\hline \multicolumn{4}{|l|}{ Aminoglycosides } \\
\hline Gentamicin & 100 & 24 & 40 \\
\hline Tobramycin & 75 & 6 & 20 \\
\hline Amikacin & 100 & 76 & 100 \\
\hline \multicolumn{4}{|l|}{ Others } \\
\hline Tetracycline & 75 & 21 & 0 \\
\hline Trimethoprim/sulphamethoxazole & 75 & 33 & 20 \\
\hline Fosfomycin & 50 & 88 & 40 \\
\hline
\end{tabular}




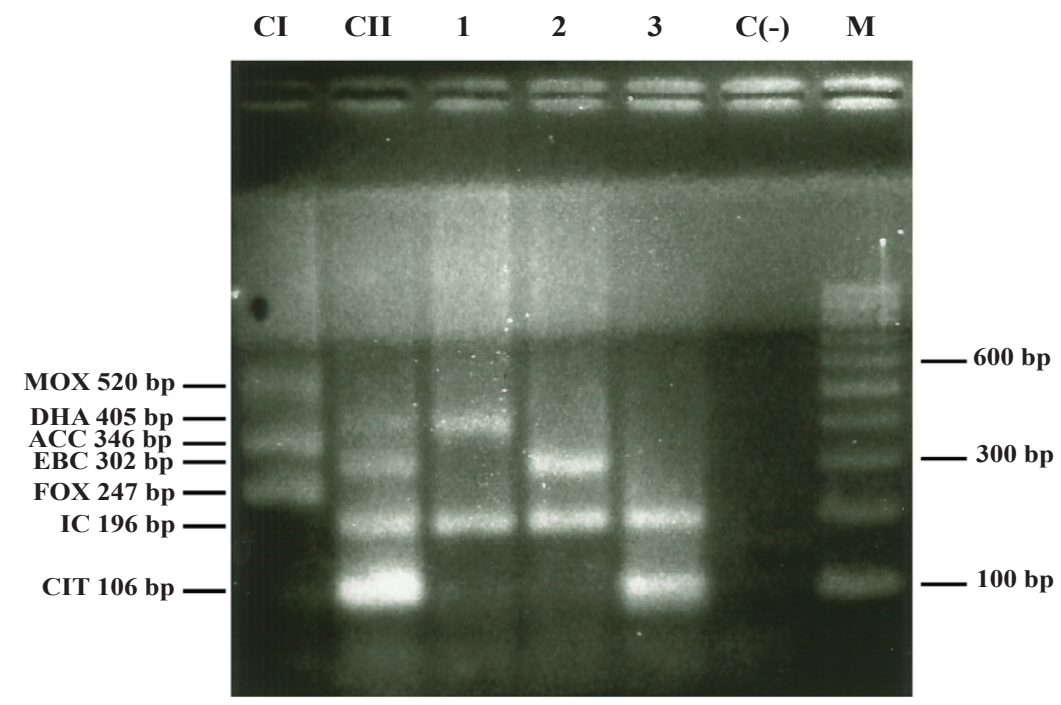

Figure 1. Multiplex PCR for ampC gene family. Lane C I: Positive control I: MOX (520 bp), ACC (346 bp), FOX (247 bp). Lane C II: Positive control II: DHA (405 bp), EBC (302 bp), CIT (160 bp). Lane C (-): Negative control. Lane 1, 2, 3: Samples with DHA positive, EBC positive and CIT positive respectively. Lane M: Standard DNA ladder for molecular size. MOX, DHA, ACC, EBC, FOX, and CIT are ampC gene gamily (DHA: firstly isolated in Dhahran Hospitals Saudi Arabia, EBC: isolated from Enterobacter cloacae, CIT: firstly isolated from Citrobacter freundii, ACC: Ambler class C, FOX: Active on Cefoxitin, and MOX: Active on Moxalactam, IC: internal control). decreased susceptibility to Meropenem. This results might suggest that these microbes carry another resistance mechanism, such as porin loss.(6) Porins play a critical role in the penetration of antibiotics into the cells, and the loss of porins can reduce susceptibility to cephalosporins and carbapenem. Porins loss are associated with boosting the resistance to carbapenem by means of ESBL and AmpC $\beta$-lactamases.(16)

Up until today, studies about molecular epidemiology of resistance pathogens in Indonesia were still limited. To our knowledge, our study was the first investigation about the gene family of AmpC-producing Enterobacteriaceae in Indonesia. Using the multiplex PCR, DHA, EBC and CIT gene families of the AmpC-producing were identified. The DHA gene family seemed to be more common than EBC and CIT. Our results were in agreement with other studies carried in India that found the CIT and DHA gene families.

Table 3. Detection of gene family of AmpCproducing Enterobacteriaceae from 6 isolates phenotypically confirmed by DDS test.

\begin{tabular}{|c|c|c|}
\hline Gene Family & DDS Test & $\begin{array}{c}\text { Enterobacteriaceae } \\
\text { Species }\end{array}$ \\
\hline \multirow{3}{*}{ DHA } & AmpC/ESBL & Enterobacter cloacae \\
\hline & AmpC/ESBL & Klebsiella pneumoniae \\
\hline & $\mathrm{AmpC}$ & Klebsiella pneumoniae \\
\hline \multirow{2}{*}{$\mathrm{EBC}$} & AmpC & Enterobacter cloacae \\
\hline & AmpC & Enterobacter aerogenes \\
\hline CIT & AmpC/ESBL & Escherichia coli \\
\hline Not detected & AmpC/ESBL & Klebsiella pneumoniae \\
\hline Not detected & AmpC/ESBL & Enterobacter cloacae \\
\hline Not detected & AmpC & Enterobacter cloacae \\
\hline
\end{tabular}

$(9,10,11)$ In Singapore, the presence of CIT gene family was reported while the EBC and DHA gene families have been found in Malaysia.(10,17) Other reports from Thailand (18) and South Korea (12) showed the presence of CIT, MOX, DHA and CIT respectively.

Hansen, et al., 2012 identified DHA gene family of AmpC-producing K. pneumoniae, however, the $\beta$-lactamase was not detected.(19) This condition could have occurred since DHA genes are inducible by $\beta$-lactam antibiotics, instead of expressed naturally.(6) Therefore the use of $\beta$-lactam antibiotics must be prudent to avoid an expression or even over expression of the $\operatorname{ampC}$ plasmid-mediated gene. In this study, $а т p C$ genes were only detected in 6 isolates out of 9 confirmed AmpC and AmpC/ESBL producers. This result could be due to the fact that of ampC gene family continue to expand, while the primers used in this study were only designated to these 6 ampC gene families. In addition, they also exist as varian i.e., the varian for the DHA are DHA-1 and DHA-2, the CIT are CMY2 and CMY-4, the EBC are ACT- 1 and MIR-1 and many more. There were some constraints in the present study, among other $\beta$-lactamase standard microorganisms for the DDS test was not available. Number of AmpC and AmpC/ ESBL isolates positive from the DDS test was too low, which then might not show the presence of all ampC gene family has ever reported by PCR.

\section{Conclusion}

This study showed the AmpC and AmpC/ESBL-producing Enterobacteriaceae were simultaneously co-exist in the ICU of Cipto Mangunkusumo Hospital. Three ampC gene families, DHA, EBC and CIT were identified. All of the 
AmpC/ESBL-producing isolates showed resistance to almost all antibiotic tested except for Amikacin. Therefore, the use of antibiotics for the treatment of the patients with infection in the ICU must be prudent to prevent the increase and the spread of the multi-drug resistant bacteria.

\section{Acknowledgements}

We would like to thank dr. Yulia R. Saharman, Clinical Microbiologist, for providing Total ESBL + AmpC kit 98019 (ROSCO Diagnostica) from Erasmus MC, Rotterdam. This work was supported by Cipto Mangunkusumo Hospital research grant 2015.

\section{References}

1. Jean SS, Hsueh PR. High burden of antimicrobial resistance in Asia. Int J Antimicrob Agents. 2011; 37: 291-5.

2. Sudarmono P. Bacteria fight back, also in Indonesia. Acta Med Indones. 2013; 45: 1-2.

3. Radji M, Fauziah S, Aribinuko N. Antibiotic sensitivity pattern of bacterial pathogens in the intensive care unit of Fatmawati Hospital, Indonesia. Asian Pac J Trop Biomed. 2011; 1: 39-42.

4. Karuniawati A, Saharman YR, Lestari DC. Detection of carbapenemase encoding genes in Enterobacteriace, Pseudomonas aeruginosa, and Acinetobacter baumanii isolated from patients at Intensive Care Unit Cipto Mangunkusumo Hospital in 2011. Acta Med Indones. 2013; 45: 101-6.

5. Bush K, Jacoby GA. Updated functional classification of B-Lactamases. Antimi-crob Agents Chemother. 2010; 54: 969-76.

6. Jacoby GA. AmpC beta-lactamases. Clin Microbiol Rev. 2009; 22: 161-82

7. Pérez-Pérez FJ, Hanson ND. Detection of plasmid-mediated AmpC beta-lactamase genes in clinical isolates by using multiplex PCR. J Clin Microbiol. 2002; 40: 2153-62.

8. Manoharan AC, Sugumar M, Kumar A, Jose H, Mathai DC. Phenotypic \& molecular characterization of AmpC $\beta$-lactamases among Escherichia coli, Klebsiella spp. \& Enter-obacter spp. from five Indian Medical Centers. Indian J Med Res. 2012; 135: 359-64.
9. Gupta V, Kumarasamy K, Gulati N, Garg R, Krishnan P, Chander J. AmpC $\beta$-lactamases in nosocomial isolates of Klebsiella pneumoniae from India. Indian J Med Res. 2012; 136: 237-41.

10. Mohamudha PR, Harish BN, Parija SC. Molecular description of plasmid-mediated AmpC $\beta$-lactamases among nosocomial isolates of Escherichia coli \& Klebsiella pneu-moniae from six different hospitals in India. Indian J Med Res. 2012; 135: 114-9.

11. Yilmaz NO, Agus N, Bozcal E, Oner O, Uzel A. Detection of plasmidmediated AmpC $\beta$-lactamase in Escherichia coli and Klebsiella pneumoniae. Indian J Med Microbiol. 2013; 31: 53-9.

12. Lee $\mathrm{CH}$, Lee YT, Kung $\mathrm{CH}, \mathrm{Ku}$ WW, Kuo SC, Chen TL, et al. Risk factors of community-onset urinary tract infections caused by plasmid-mediated AmpC $\beta$-lactamase-producing Enterobacteriaceae. J Microbiol Immunol Infect. 2015; 48: 26975.

13. Leverstein-van Hall MA, Blok HEM, Paauw A, Fluit AC, Troelstra A, Mascini EM, et al. Extensive hospital-wide spread of a multidrugresistant Enterobacter cloacae clone, with late detection due to a variable antibiogram and frequent patient transfer. J Clin Microbiol. 2006; 44: 518-24.

14. Clinical and Laboratory Standard Institute (CLSI). Performance Standard for Antimicro-bial Susceptibility Testing: 24th Informational Supplement. CLSI document M100-S24. Wayne, PA: CLSI; 2014.

15. Tan TY, Ng LS, He J, Hsu LY. CTX-M and ampC beta-lactamases contributing to increased prevalence of ceftriaxone-resistant Escherichia coli in Changi General Hospital, Singapore. Diagn Microbiol Infect Dis. 2010; 66: 210-3.

16. Shi W, Li K, Ji Y, Jiang Q, Wang Y, Shi M, et al. Carbapenem and cefoxitin resistance of Klebsiella pneumoniae strains associated with porin OmpK36 loss and DHA-1 $\beta$-lactamase production. Braz J Microbiol. 2013; 44: 435-42.

17. Mohd Khari FI, Karunakaran R, Rosli R, Tee Tay S. Genotypic and phenotypic detection of AmpC $\beta$-lactamases in Enterobacter spp. isolated from a teaching hospital in Malaysia. PLoS One. 2016; 11: e0150643. doi:10.1371/journal.pone.0150643.

18. Singtohin S, Chanawong A, Lulitanond A, Sribenjalux P, Auncharoen A, Kaewkes W, et al. CMY-2, CMY-8b, and DHA-1 plasmidmediated AmpC $\beta$-lactamases among clinical isolates of Escherichia coli and Klebsiella pneumoniae from a university hospital, Thailand. Diagn Microbiol Infect Dis. 2010; 68: 271-7.

19. Hansen F, Hammerum AM, Skov RL, Giske CG, Sundsfjord A, Samuelsen O. Evaluation of ROSCO Neo-Sensitabs for phenotypic detection and subgrouping of ESBL-, AmpC- and carbapenemaseproducing Enterobacteriaceae. APMIS. 2012; 120: 724-32. 